\title{
Improved salt tolerance of Chenopodium quinoa Willd. contributed by Pseudomonas sp. strain M30-35
}

\author{
Deyu Cai ${ }^{1,2,3}$, Ying Xu ${ }^{2}$, Fei Zhao ${ }^{1}$, Yan Zhang ${ }^{2}$, Huirong Duan ${ }^{\text {Corresp., } 4}$, Xiaonong Guo ${ }^{\text {Corresp. 1, 2, } 3}$ \\ ${ }^{1}$ Key Laboratory of Biotechnology and Bioengineering of State Ethnic Affairs Commission, Biomedical Research Center, Northwest Minzu University, \\ Lanzhou, China \\ 2 College of life science and engineering, Northwest Minzu University, Lanzhou, China \\ 3 China-Malaysia National Joint Laboratory, Biomedical Research Center, Northwest Minzu University, Lanzhou, China \\ 4 Lanzhou Institute of Husbandry and Pharmaceutical Sciences, Chinese Academy of Agricultural Sciences, Lanzhou, China \\ Corresponding Authors: Huirong Duan, Xiaonong Guo \\ Email address: duanhuirong@caas.cn, gxnwww@xbmu.edu.cn
}

Background. Plant-growth-promoting rhizobacteria (PGPR) can promote plant growth and enhance plant tolerance to salt stress. Pseudomonas sp. strain M30-35 might confer abiotic stress tolerance to its host plants. We evaluated the effects of M30-35 inoculation on the growth and metabolite accumulation of Chenopodium quinoa Willd. during salt stress growth conditions.

Methods. The effects of M30-35 on the growth of $C$. quinoa seedlings were tested under salt stress. Seedling growth parameters measured included chlorophyll content, root activity, levels of plantphosphorus $(\mathrm{P})$, and saponin content.

Results. M30-35 increased biomass production and root activity compared to non-inoculated plants fertilized with rhizobia and plants grown under severe salt stress conditions. The photosynthetic pigment content of chlorophyll $a$ and $b$ were higher in M30-35-inoculated C. quinoa seedlings under high salt stress conditions compared to non-inoculated seedlings. The stability of $\mathrm{P}$ content was also maintained. The content of saponin, an important secondary metabolite in C. quinoa, was increased by the inoculation of M30-35 under $300 \mathrm{mM} \mathrm{NaCl}$ conditions.

Conclusion. Inoculation of M30-35 rescues the growth diminution of $C$. quinoa seedlings under salt stress. 


\section{Improved salt tolerance of Chenopodium quinoa Willd.} 2 contributed by Pseudomonas sp. strain M30-35

3

4

5
Deyu Cai 1,2,3, Ying Xu ${ }^{2}$, Fei Zhao ${ }^{1}$, Yan Zhang ${ }^{2}$, Huirong Duan ${ }^{4}$, Xiaonong Guo 1,2,3

${ }^{1}$ Key Laboratory of Biotechnology and Bioengineering of State Ethnic Affairs Commission, Biomedical Research Center, Northwest Minzu University, Lanzhou, Gansu, China

${ }^{2}$ College of life science and engineering, Northwest MinzuUniversity, Lanzhou, China

${ }^{3}$ China-Malaysia National Joint Laboratory, Biomedical Research Center, Northwest Minzu University, Lanzhou, China

${ }^{4}$ Lanzhou Institute of Husbandry and Pharmaceutical Sciences, Chinese Academy of Agricultural Sciences,Lanzhou, China

Corresponding Author:

Huirong Duan ${ }^{4 *}$, Xiao-nong Guo ${ }^{1,2,3 *}$

Email address: duanhuirong@caas.cn (H.D.); gxnwww@126.com (X.G.) 


\section{Abstract}

41 Background. Plant-growth-promoting rhizobacteria (PGPR) can promote plant growth and enhance plant tolerance to salt stress. Pseudomonas sp. strain M30-35 might confer abiotic stress tolerance to its host plants. We evaluated the effects of M30-35 inoculation on the growth and metabolite accumulation of Chenopodium quinoa Willd. during salt stress growth conditions. Methods. The effects of M30-35 on the growth of $C$. quinoa seedlings were tested under salt stress. Seedling growth parameters measured included chlorophyll content, root activity, levels of plant- phosphorus (P), and saponin content.

Results. M30-35 increased biomass production and root activity compared to non-inoculated plants fertilized with rhizobia and plants grown under severe salt stress conditions. The photosynthetic pigment content of chlorophyll a and b were higher in M30-35-inoculated $C$. quinoa seedlings under high salt stress conditions compared to non-inoculated seedlings. The stability of P content was also maintained. The content of saponin, an important secondary metabolite in C. quinoa, was increased by the inoculation of $\mathrm{M} 30-35$ under $300 \mathrm{mM} \mathrm{NaCl}$ conditions.

Conclusion. Inoculation of M30-35 rescues the growth diminution of C. quinoa seedlings under salt stress.

57

\section{8}

59

60

61

62

63

64

65

66

67

68

69

70

71

72

73

74

75

76

77

78

\section{Introduction}

Salinity is a major threat to crop production, especially in arid and semi-arid areas (Flowers \& Colmer, 2008; Schleiff, 2008). Salt stress has a negative effect on plant growth, and development (Yu et al., 2018), and it is challenging to determine the efficient use of saline soilsfor food production. Two primary strategies, modified agricultural practices and the development of new varieties adapted to saline soils, are being used for growing crops in saline soils. These strategies have produced useful, results but they are still in the early stages of development (Panta et al., 2014). The use of plant-growth-promoting rhizobacteria (PGPR) may help enhance plant stress resistance (Elesawi et al., 2018; Gururani \& Upadhyay, 2013; Kuzyakov \& Razavi, 2019).

PGPR may improve safe crop-management practices and stress resistance (Tiwari \& Lata, 2018). Kloepper and Schroth (1978) proposed the concept of PGPR, which can promote plant growth and crop yield and improve plant resistance to biotic or abiotic stress. PGPR are also important in reducing oxidative damage and the negative effects of abiotic stresses such as drought, extreme temperatures and heavy metal stress (Bresson et al., 2013; Gururani \& Upadhyay., 2013; Khan et al., 2019; Vardharajula et al., 2011). Pseudomonas sp. M30-35 is a novel PGPR strain isolated from the rhizosphere of Haloxylon ammodendron (C.A.Mey.) Bunge (Chenopodiaceae) in the Tengger desert of northern China. M30-35 inoculation significantly improved the growth performance of perennial ryegrass (Lolium perenne L.) under high salt stress (He et al., 2018). Biofertilizer containing M30-35 improved the efficacy of biofertilizer alone (Gou et al., 2020). It is not known if M30-35 is associated with protective beneficial effects on halophytes. Hence, further studies are needed. 
79

80

81

82

83

84

85

86

87

88

89

90

91

92

93

94

95

96

97

98

99

100

101

102

103

104

105

106

107

108

109

110

111

112

113

114

115

116

Chenopodium quinoa is a perennial halophyte that originated in the Andes Mountains of South America and has been cultivated for more than 7,000 years (Jacobsen et al., 2009). C. quinoa grows well under moderate salt stress $(100-200 \mathrm{mM}$ of $\mathrm{NaCl})$, and can withstand a concentration of $400 \mathrm{mM}$ of $\mathrm{NaCl}$ (Jacobsen et al., 2003). C. quinoa is rich in proteins, amino acids, dietary fibers, vitamins, minerals, and beneficial phytochemicals such as saponins (Navruz-Varli \& Sanlier, 2016). Thus, C. quinoa is an ideal plant for evaluating the effects of PGPR on plant salt tolerance. However, the roles of PGPR on the salt resistance modulation of C. quinoa may be limited.

In the present study, the effects of M30-35 on the growth and salt tolerance of C. quinoa were analyzed under different salt treatments, and the physiological responses were compared.

\section{Materials \& Methods}

Bacterial strain culture.

Luria broth (LB) $[5 \mathrm{~g} / \mathrm{L}$ yeast extract (Solarbio, Beijing, China), $10 \mathrm{~g} / \mathrm{L}$ peptone (Solarbio, Beijing, China), $10 \mathrm{~g} / \mathrm{L}$ sodium chloride (Guangfu, Tianjin, China), PH 7.2] were used for bacterial culture. Pseudomonas sp. strain M30-35 (obtained from the State Key Laboratory of Grassland Agro-Ecosystems, College of Pastoral Agriculture Science and Technology, Lanzhou University, Lanzhou, China; the strain is deposited in the Marine Culture Collection of China with preservation number $1 \mathrm{k} 03247$.) was incubated, in the dark, at $28^{\circ} \mathrm{C}$, with shaking at $220 \mathrm{rpm}$ (YiHeng, THZ-98AB, ShangHai, China), for $24 \mathrm{~h}$. Bacteria were harvested at an $\mathrm{OD}_{600}$ between 0.9 and 1.0 , then diluted to $10^{9}$ colony forming units (CFU) $\mathrm{mL}^{-1}$, as measured by optical density and serial dilutions (Zhao et al., 2016).

\section{Plant growth and treatments}

The C. quinoa seeds (Animal Husbandry, Pasture and Green Agriculture Institute, GAAS, Gansu, China) were surface sterilized with $0.5 \%$ potassium permanganate solution for $10 \mathrm{~min}$ and washed with sterile water until colorless (Zheng et al., 2017). The seeds were immersed in LB liquid medium (blank control) and Pseudomonas sp. M30-35 bacterial solution (treatment group) for $20 \mathrm{~min}$. Then, the $C$. quinoa seeds were germinated on moist filter paper in Petri dishes and kept for $48 \mathrm{~h}$ at $25^{\circ} \mathrm{C}$ in darkness (Gururani \& Upadhyaya, 2013). After germination, the plantlets were transferred to plastic pots (upper diameter, $48 \mathrm{~mm}$; bottom, $23 \mathrm{~mm}$; depth, 50 $\mathrm{mm}$ ). The pots contained vermiculite, which was previously heated at $121^{\circ} \mathrm{C}$ for $20 \mathrm{~min}$ for sterilization. Each pot was inoculated with $1 \mathrm{~mL}$ of M30-35 suspension culture or $1 \mathrm{~mL}$ of liquid LB medium. Each treatment was irrigated with half-strength Hoagland solution $\left(2 \mathrm{mM} \mathrm{KNO}_{3}\right.$, $0.5 \mathrm{mM} \mathrm{NH}_{4} \mathrm{H}_{2} \mathrm{PO}_{4}, 0.5 \mathrm{mM} \mathrm{Ca}\left(\mathrm{NO}_{3}\right)_{2}, 0.5 \mathrm{mM} \mathrm{MgSO}_{4}, 0.5 \mathrm{mM}$ Fe-citrate, $92 \mu \mathrm{M} \mathrm{H}_{3} \mathrm{BO}_{3}, 18$ $\mu \mathrm{M} \mathrm{MnCl}_{2} \cdot 4 \mathrm{H}_{2} \mathrm{O}, 1.6 \mu \mathrm{M} \mathrm{ZnSO}_{4} \cdot 7 \mathrm{H}_{2} \mathrm{O}, 0.6 \mu \mathrm{M} \mathrm{CuSO}_{4} \cdot 5 \mathrm{H}_{2} \mathrm{O}$, and $0.7 \mu \mathrm{M}$ $\left.\left(\mathrm{NH}_{4}\right)_{6} \mathrm{Mo}_{7} \mathrm{O}_{24} \cdot 4 \mathrm{H}_{2} \mathrm{O}\right)(2 \mathrm{~L})$. The plants were grown in a greenhouse with a $16: 8 \mathrm{~h}(\mathrm{~L}: \mathrm{D})$ photoperiod, $26^{\circ} \mathrm{C}$ day and $22^{\circ} \mathrm{C}$ night temperature, and a relative humidity of $65 \pm 10 \%$ (He et al., 2018). 
117

118

119

120

121

122

123

124

125

126

127

128

129

130

131

132

133

134

135

136

137

138

139

140

141

142

143

144

145

146

147

148

149

150

151

152

153

154

155

156

157

Plants aged $14 \mathrm{~d}$ were subjected to salt stress by treatment with 0,150 , or $300 \mathrm{mM}$ of $\mathrm{NaCl}$ solutions. Treated seedlings were harvested at $7 \mathrm{~d}$ and $21 \mathrm{~d}$ after salt treatment to record the plant biomass and physiological measurements (Su et al., 2017).

\section{Determination of the seedling biomass}

Plants were removed from the pots, and the roots were washed with sterilized water to remove attached soil. Then, the seedlings were divided into shoot and root portions. The samples were dried in an oven at $70^{\circ} \mathrm{C}$ for $4 \mathrm{~d}$, and the dry weight was measured (Elesawi et al., 2018).

\section{Determination of chlorophyll contents}

The relative chlorophyll content was measured using the ethanol-acetone method, according to the method previously reported (Han et al., 2014). Chlorophyll was extracted from the third compound leaf $(50 \mathrm{mg}$ ) with an $80 \%(\mathrm{v} / \mathrm{v})$ cold acetone and $95 \%$ ethyl alcohol mix (1:1). A spectrophotometer (Shimadzu, UV-1800, Suzhou, China) was used to read the absorbance at 663 $\mathrm{nm}$ for chlorophyll a and $645 \mathrm{~nm}$ for chlorophyll b contents. Six samples were measured in each treatment. Each measurement was conducted at 10:00 a.m.

\section{Determination of root activity}

Root activity was determined using the triphenyltetrazolium chloride (TTC) colorimetric method (Muhammad et al., 2019). C. quinoa seedling roots were washed, and the root tip samples were excised. Then, the root tips were dried with filter paper. The reaction mixture consisted of $0.2 \mathrm{~g}$ root samples, $5 \mathrm{~mL}$ of PBS (pH 7.0), and $5 \mathrm{~mL}$ of $0.4 \%$ TTC in a tube, with the root tips fully immersed in the solution for $2 \mathrm{~h}$ at $37^{\circ} \mathrm{C}$. A $2 \mathrm{~mL}$ amount of $1 \mathrm{M}$ of sulfuric acid was added to stop the reaction. The roots were removed from the reaction solution, blotted, and homogenized using a mortar and pestle. The red extraction was collected by ethyl acetate to determine the final volume of $10 \mathrm{~mL}$. The absorbance was determined at $485 \mathrm{~nm}$ against a blank of ethyl acetate. The standard curve reduction value was TTC $(\mathrm{y})$ : $\mathrm{y}=0.0021 \mathrm{x}-0.0113, \mathrm{R}=0.9945$, where $\mathrm{y}$ represents the absorbance reading. The root vigor $=\mathrm{TTC}(\mathrm{x}) / \mathrm{W} \times$ time reduction value, where $\mathrm{W}$ is the root fresh weight, and the time was $2 \mathrm{~h}$.

\section{Determination of total $\mathbf{P}$ content}

Phosphorus content was determined by the molybdenum blue spectrophotometry method (Tisarum et al., 2020). Dried shoot and root samples (100 mg) were added to $8 \mathrm{~mL}$ of $\mathrm{H}_{2} \mathrm{SO}_{4}$ and soaked overnight. Then, this was digested at $300^{\circ} \mathrm{C}$ for $90 \mathrm{~min}$ until fumes were produced. After slight cooling, $0.5 \mathrm{~mL}$ of $\mathrm{H}_{2} \mathrm{O}_{2}$ was added to the tubes, and heated at $300^{\circ} \mathrm{C}$ until the solution was colorless or clear. The volume of extract was maintained by adding distilled water up to 100 $\mathrm{mL}$ in the volumetric flask. Then, $10 \mathrm{~mL}$ of the previous digestion liquid was transferred to a 50 $\mathrm{mL}$ volumetric flask, and dinitrophenol indicator was added. Afterwards, $5 \mathrm{~mL}$ of molybdenum antimony anti-coloring agent was added, shaken well, and added with up to $50 \mathrm{~mL}$ of ultrapure water. Total $\mathrm{P}\left(\mathrm{mg} \mathrm{g}^{-1} \mathrm{DW}\right)$ was measured at $420 \mathrm{~nm}$ by a spectrophotometer using $\mathrm{KH}_{2} \mathrm{PO}_{4}$ as the calibration standard.

\section{Saponin quantification}

The content of total saponin in the different salt treatments of $C$. quinoa was determined by colorimetry (Liao et al., 2017) using oleanolic acid as the standard. C. quinoa saponins were retrieved by microwave heating. The dried shoot and root samples $(500 \mathrm{mg})$ were extracted with 
158

159

160

161

162

163

164

165

166

167

168

169

170

171

172

173

174

175

176

177

178

179

180

181

182

183

184

185

186

187

188

189

190

191

192

193

194

195

196

197

$10 \mathrm{~mL}$ of $75 \%$ alcohol placed in a ultrasonic bath for $30 \mathrm{~min}$ at $60^{\circ} \mathrm{C}$. The extract solution was then evaporated to dryness in $60^{\circ} \mathrm{C}$ water. Next, both the $200 \mu \mathrm{L} 5 \%$ vanillin acetic acid (vanillin: glacial acetic acid was $5: 100, \mathrm{~W} / \mathrm{V}$ ) and $800 \mu \mathrm{L}$ perchloric acid were added to different test tubes. The liquid was boiled for $15 \mathrm{~min}$, and up to $5 \mathrm{~mL}$ of glacial acetic acid was added in a volumetric flask after cooling. The absorbance was determined at $546 \mathrm{~nm}$ using a spectrophotometer against the reagent blank.

\section{Data Analysis}

Excel 2010 was used to collect and process the original data. All data were analyzed by one-way analysis of variance (ANOVA). Duncan's post-hoc multiple comparison tests were used to identify differences between means at a significance level of $P \leq 0.05$ using SPSS version 21.0 (IBM Corporation) statistical software. Results of the growth and physiological data are presented as means \pm standard error. Data were plotted using the ORIGIN software.

\section{Results}

\section{M30-35 effects on C. quinoa growth}

With increased salinityfor $7 \mathrm{~d}$ and $21 \mathrm{~d}$, the dry weight of shoots decreased in the control and the M30-35 medium. For the $7 \mathrm{~d}$ treatment, different $\mathrm{NaCl}$ concentrations had no effect on the shoot dry weight of the control, while $150 \mathrm{mM}$ of $\mathrm{NaCl}$ increased the shoot dry weight of seedlings inoculated with M30-35 (Fig. 1A). For the $21 \mathrm{~d}$ treatment, M30-35 and the control had similar levels of reduced growth. In contrast, M30-35 reduced the decline of shoot dry weight (Fig. 1A and 1B). M30-35 slightly improved shoot growth, compared to the control, under $\mathrm{NaCl}$ treatment.

We compared the effect of adding Luria broth (LB) to plants grown in the presence or absence of M30-35. The roots maintained normal growth after $7 \mathrm{~d}$ in the $\mathrm{NaCl}$ treatment. However, roots were reduced by exposure to $\mathrm{NaCl}$ for $21 \mathrm{~d}$. Compared to the control, M30-35 significantly improved the dry weight of roots by $56.67 \%$ and $51.97 \%$ at 0 and $150 \mathrm{mM} \mathrm{NaCl}$ treatments for $7 \mathrm{~d}$. After $21 \mathrm{~d}, \mathrm{M} 30-35$ provided a non-significant improvement in the dry weight of roots under salt treatment. The seedlings inoculated with M30-35 had increased root dry weights under different $\mathrm{NaCl}$ treatments (Fig. 1C and 1D).

\section{Influence of M30-35 on the chlorophyll content of C. quinoa}

After a prolonged time period, both chlorophyll a and chlorophyll b in C. quinoa seedlings showed similar changes under the $\mathrm{NaCl}$ treatments (Fig. 2). After $\mathrm{NaCl}$ treatment for $7 \mathrm{~d}$, the chlorophyll a and chlorophyll $\mathrm{b}$ levels in the controls significantly decreased with the increase in salt concentrations. However, the levels of chlorophyll a and b in the M30-35 treatment maintained relative stability (Fig. $2 \mathrm{~A}$ and $2 \mathrm{C}$ ). Under $300 \mathrm{mM} \mathrm{NaCl}$ conditions the chlorophyll a content of the M30-35 treatment significantly increased by $40.48 \%$, compared to the controls $(P<0.05)$. In contrast, the content of chlorophyll a and $\mathrm{b}$ in the control or M30-35 medium in the $\mathrm{NaCl}$ treatment at $21 \mathrm{~d}$ remained stable except for a significant increase in chlorophyll $\mathrm{b}$ (66.54\%) under the $300 \mathrm{mM} \mathrm{NaCl}$ treatment (Fig. 2B and 2D).

\section{Impact of M30-35 on the root activity of $C$. quinoa}


198 The activity of root system is related to the survival rate of plant, which is an important index to

199

200

201

202

203

204

205

206

207

208

209

210

211

212

213

214

215

216

217

218

219

220

221

222

223

224

225

226

227

228

229

230

231

232

233

234

235

236

237

reflect the quality of plant seedlings and evaluate the response of plants to stress (Zhang et al., 2019). With increasing $\mathrm{NaCl}$ concentration, the $C$. quinoa root activity in both the control and

M30-35 medium improved at $150 \mathrm{mM}$, but subsequently declined by $300 \mathrm{mM}$, at both $7 \mathrm{~d}$ and 21 d (Fig. 3). Compared to the control group, the root activity of seedlings inoculated with M30-35 improved by $11.88 \%(P<0.05)$ at $150 \mathrm{mM} \mathrm{NaCl}$ for $7 \mathrm{~d}$, and improved by $27.12 \%(P<0.05)$ under $300 \mathrm{mM}$ of $\mathrm{NaCl}$ for $21 \mathrm{~d}$.

\section{The effect of $\mathrm{M} 30-35$ on the total $P$ contents of $C$. quinoa}

The $\mathrm{P}$ content of all LB-treated plants were reduced after exposure to salt stress (Fig. 4). The inoculation of M30-35 maintained a relatively stable P content in plants under salt stress. The P content of the M30-35 treatment was significantly higher than that in the controls under $150 \mathrm{mM}$ of $\mathrm{NaCl}$ for $7 \mathrm{~d}$ (Fig. 4A).

\section{Effect of M30-35 on the saponin content in C. quinoa seedlings}

Similar to the change pattern of root activity, the total saponin content initially increased and then decreased. In the $300 \mathrm{mM} \mathrm{NaCl}$ treatment, M30-35 significantly increased the saponin content by $17.40 \%(P<0.05)$. However, M30-35 had no significant effect on the saponin content in the $150 \mathrm{mM} \mathrm{NaCl}$ treatment (Fig. 5).

\section{Discussion}

PGPR can improve the salt tolerance of plants and also promote their growth and development (Safdarian et al., 2019). Pseudomonas includes many common species that can colonize plant roots (Han et al., 2014). These strains can have significant interactions with host plants and induce plant growth (Egamberdieva et al., 2017; Han et al., 2014). Under salt stress, PGPR can increase the root length, root surface area, and number of root tips, thereby enhancing the uptake of plant nutrition, and ultimately improving plant growth and development under stress (Egamberdieva \& Kucharova, 2009; Trivedi et al., 2020). Roots colonized by the M30-35 strain enhanced the tolerance of the whole plant to salt stress, and the enhancement was reflected by increasing plant root growth and nutritional status (He et al., 2018). Halotolerant strains of bacteria (Enterobacter sp. [MN17] and Bacillus sp. [MN54]) can promote the plant health and performance of C. quinoa (Yang et al., 2016). The present results also demonstrated that M30-35 significantly increased the and dry weight of roots under saline conditions and also increased the fresh dry weight of the shoots. Serratia liquefaciens KM4 promoted maize (Zea mays L.) salt tolerance by inducing the accumulation of the biomass yield (Elesawi et al., 2018). M30-35 might improve the salt tolerance of $C$. quinoa in a similar manner. However, this hypothesis requires further verification.

Photosynthesis is the basis for increasing in plant biomass and provides the energy needed for metabolism. Changes in chlorophyll levels can be considered to be a biochemical marker of salt tolerance, which reflects the physiological state of a plant's response to salt (Bernal-Vicente et al., 2018; Singh \& Gautam, 2013). Therefore, chlorophyll plays key roles in plant development and stress resistance processes, and its concentration has been shown to decline less 
238 rapidly in strong stress-resistant plants, when compared to stress-sensitive plants (Liu \& Jiang, 239 2010). In the present study, the application of M30-35 reduced the decline of chlorophyll a and b 240 in the leaves of $C$. quinoa seedlings after $7 \mathrm{~d}$ of $\mathrm{NaCl}$ treatment, indicating the potential role of 241 M30-35 in protection against salt stress. In the $300 \mathrm{mM} \mathrm{NaCl}$ treatment, seedlings inoculated 242 with M30-35 had significantly increased chlorophyll a content after the one-week treatment, and 243 chlorophyll $\mathrm{b}$ content in the $7 \mathrm{~d}$ treatment, and increased chlorophyll b levels after the $21 \mathrm{~d}$

244

245

246

247

248

249

250

251

252

253

254

255

256

257

258

259

260

261

262

263

264

265

266

267

268

269

270

271

272

273

274

275

276 treatment. These results suggest that M30-35 could maintain the C. quinoa chlorophyll content, especially when seedlings were under severe salt stress. These results were similar to observations made on white clover and ryegrass (Han et al., 2014; He et al., 2018).

The plant root system is among the most important components of plants, and is sensitive to salt stress (Liu et al., 2019). Salt stress can reduce root vigor which was defined as the capacity for deoxidization, and affect absorption of water and nutrition (He et al., 2018). However, increased antioxidant substance accumulation and increased antioxidant enzyme activities can protect plants against the oxidative injury caused by salt stress, thereby giving the plants increased resistance to salt stress (Liu et al., 2019; Wang et al., 2014). Enzymes such as superoxide dismutase in the root of Kandelia candel under varying salt stresses exhibited a trend from ascent to descent (Wang et al., 2014). The root activity of C. quinoa was significantly greater under the $150 \mathrm{mM} \mathrm{NaCl}$ treatment, compared to the control. The higher accumulation of antioxidant substances was likely due to PGPR-induced salt stress response strategy in plants. This observation suggests that the $150 \mathrm{mM} \mathrm{NaCl}$ concentration is a moderate salt treatment for C. quinoa. Significantly elevated root activity in the M30-35 treatment was also observed under 150 and $300 \mathrm{mM}$ of $\mathrm{NaCl}$. In a similar study, Trichoderma longibrachiatum T6 significantly increased the root activity of wheat seedlings under salt stress (Zhang et al., 2019). According to the recent research results reported by He et al. (2018), M30-35 promoted the growth of ryegrass, by enhancing root activity under salt stress. Hence, the growth promotion in C. quinoa roots might be correlated to an increase in root activity under salt stress.

Salinity interferes with the absorption of macro-elements ( $\mathrm{K}, \mathrm{Ca}, \mathrm{Mg}, \mathrm{P}$, and $\mathrm{S}$ ) and microelements $(\mathrm{Zn}, \mathrm{Fe}, \mathrm{Mn}, \mathrm{Cu}$, and $\mathrm{B})$ by plants, leading to nutrient deficiency and metabolic disorders (Egamberdieva et al., 2017; Kim et al., 2017; Munns \& Tester, 2008). P is a key nutrient element in plants and plays an important role in the process of plant growth and development. The solubilization of bound inorganic $\mathrm{P}$ and mobilization of organic $\mathrm{P}$ by PGPR are mechanisms that have a positive effect on plant growth (Liu et al., 2014; Zeng et al., 2016). Under high salt conditions, eggplant seedlings inoculated with Achromobacter increased the absorption of P (Mayak et al., 2004), and inoculated wheat seedlings with Bacillus increased the levels of P in seedlings (Upadhyay \& Singh, 2015). Our results showed that the P content of noninoculated C. quinoa seedlings under salt stress was significantly lower than seedlings not exposed to salt stress. This indicated that salt stress can influence $\mathrm{P}$ absorption. The results of the present study indicate that inoculation of M30-35 can maintain $(\mathrm{P})$ homeostasis and mitigate the salt stress impact on C. quinoa. 
277

278

279

280

281

282

283

284

285

286

287

288

289

290

291

292

293

294

295

296

297

298

299

300

301

302

303

304

305

306

307

308

309

310

311

312

313

314

315

316

317

318

C. quinoa is rich in saponins (belonging to the triterpenoid saponins) mainly concentrated in the pericarp (Kuljanabhagavad et al., 2008). The saponins contents vary in different C. quinoa varieties and are in the range of 47.11-136.98 mg/100g, which are often influenced by biotic and abiotic factors (Szakiel et al., 2011). Under $200 \mathrm{mM}$ of salt stress, the C. quinoa seedlings accumulated higher levels of saponins than control (Gomez-Caravaca et al., 2012). Furthermore, Yang et al. (2018) demonstrated that saponins might be a biostimulant for germination in $C$. quinoa, especially under salt stress. Thus, saponin might be involved in the response of $C$. quinoa to salt stress. Our results showed that $150 \mathrm{mM}$ of $\mathrm{NaCl}$ induced the synthesis of saponins in C. quinoa seedlings. Besides, the C. quinoa plants inoculated with M30-35 exhibited higher saponins levels compared to un-inoculated plants under $300 \mathrm{mM} \mathrm{NaCl}$ salt stress. M30-35 might enhance the salt tolerance of C.quinoa by partially increasing their saponins accumulation.

\section{Conclusions}

The effects of Pseudomonas sp. strain M30-35 on the growth and physiology of C. quinoa seedlings were evaluated. Under salt stress, M30-35 promoted the growth of C. quinoa, and increased the root and total biomass of $C$. quinoa seedlings. The chlorophyll a and chlorophyll $\mathrm{b}$ content in the $300 \mathrm{mM} \mathrm{NaCl}$ treatment was increased by inoculation of M30-35. M30-35 improved the root activity and accumulation of total saponins and alleviated the decline in $\mathrm{P}$ content under salinity. These data provide evidence that M30-35 inoculation of C. quinoa seedlings can improve their salt tolerance.

\section{Acknowledgements}

This research was supported by National Natural Science Foundation of China (31760242) and Characteristic discipline of bioengineering construction for the special guide project of the "world-class universities and world-class disciplines" of Northwest Minzu University (1001070204, 11080306) and the Ministry of Education of China for an Innovative Research Team in University (IRT 17R88) and the Fundamental Research Funds for the Central Universities grant number (31920190021).The authors also acknowledge Dr. Zhao Qi for providing experimental materials. We thank LetPub (www.letpub.com) for its linguistic assistance during the preparation of this manuscript.

\section{References}

Bernal-Vicente A, Cantabella D, Petri C, Hernández JA, and Diaz-Vivancos P. 2018. The Salt-Stress Response of the Transgenic Plum Line J8-1 and Its Interaction with the Salicylic Acid Biosynthetic Pathway from Mandelonitrile. International Journal of Molecular Sciences 19:3519. 10.3390/ijms 19113519

Bresson J, Varoquaux F, Bontpart T, Touraine B, and Vile D. 2013. The PGPR strain Phyllobacterium brassicacearum STM196 induces a reproductive delay and physiological changes that result in improved drought tolerance in Arabidopsis. New Phytologist 200:558-569. 10.1111/nph.12383

Dixon RA. 2001. Natural products and plant disease resistance. Nature 411:843-847. 10.1038/35081178

Egamberdieva D, and Kucharova Z. 2009. Selection for root colonising bacteria stimulating wheat growth in saline soils. Biology and Fertility of Soils 45:563-571. 10.1007/s00374-009-0366-y 
319

320

321

322

323

324

325

326

327

328

329

330

331

332

333

334

335

336

337

338

339

340

341

342

343

344

345

346

347

348

349

350

351

352

353

354

355

356

357

358

359

360

361

362

363

364

365

366

367

368

Egamberdieva D, Wirth S, Jabborova D, Rasanen LA, and Liao H. 2017. Coordination between Bradyrhizobium and Pseudomonas alleviates salt stress in soybean through altering root system architecture. Journal of Plant Interactions 12:100-107. 10.1080/17429145.2017.1294212

Elesawi MA, Alaraidh IA, Alsahli AA, Alzahrani SM, Ali HM, Alayafi AA, and Ahmad M. 2018. Serratia liquefaciens KM4 Improves Salt Stress Tolerance in Maize by Regulating Redox Potential, Ion Homeostasis, Leaf Gas Exchange and Stress-Related Gene Expression.

International Journal of Molecular Sciences 19:3310. 10.3390/ijms19113310

Faizal A, and Geelen D. 2013. Saponins and their role in biological processes in plants. Phytochemistry Reviews 12:877-893. 10.1007/s11101-013-9322-4

Flowers TJ, and Colmer TD. 2008. Salinity tolerance in halophytes*. New Phytologist 179:945-963. 10.1111/j.1469-8137.2008.02531.x

Gomezcaravaca AM, Iafelice G, Lavini A, Pulvento C, Caboni MF, and Marconi E. 2012. Phenolic compounds and saponins in quinoa samples (Chenopodium quinoa Willd.) grown under different saline and nonsaline irrigation regimens. Journal of Agricultural and Food Chemistry 60:46204627. 10.1021/jf3002125

Gou JY, Suo SZ, Shao KZ, Zhao Q, Yao D, Li HP, Zhang JL, and Rensing C. 2020. Biofertilizers with beneficial rhizobacteria improved plant growth and yield in chili (Capsicum annuum L.). World Journal of Microbiology \& Biotechnology 36. 10.1007/s11274-020-02863-w

Gururani MA, and Upadhyaya CP. 2013. Plant Growth-Promoting Rhizobacteria Enhance Abiotic Stress Tolerance inSolanum tuberosumThrough Inducing Changes in the Expression of ROSScavenging Enzymes and Improved Photosynthetic Performance. Journal of Plant Growth Regulation 32:245-258. 10.1007/978-3-7643-8554-5_1

Haghighi Z, Karimi N, Modarresi M, and Mollayi S. 2012. Enhancement of compatible solute and secondary metabolites production in Plantago ovata Forsk. by salinity stress. Journal of medicinal plant research 6:3495-3500. 10.5897/JMPR12.159

Han Q, Lu X, Bai J, Qiao Y, Pare PW, Wang S, Zhang J, Wu Y, Pang X, and Xu W. 2014. Beneficial soil bacterium Bacillus subtilis (GB03) augments salt tolerance of white clover. Frontiers in Plant Science 5:525-525. 10.3389/fpls.2014.00525

He A, Niu S, Zhao Q, Li Y, Gou J, Gao H, Suo S, and Zhang J. 2018. Induced Salt Tolerance of Perennial Ryegrass by a Novel Bacterium Strain from the Rhizosphere of a Desert Shrub Haloxylon ammodendron. International Journal of Molecular Sciences 19:469. 10.3390/ijms19020469

Jacobsen S, Liu F, and Jensen CR. 2009. Does root-sourced ABA play a role for regulation of stomata under drought in quinoa (Chenopodium quinoa Willd.). Scientia Horticulturae 122:281-287. 10.1016/j.scienta.2009.05.019

Jacobsen SE, Mujica A, and Jensen CR. 2003. The Resistance of Quinoa (Chenopodium quinoa Willd.) to Adverse Abiotic Factors. Food Reviews International 19:p.99-109. 10.1081/FRI-120018872

Khan N, Bano A, Rahman MA, Guo J, Kang Z, and Babar MA. 2019. Comparative Physiological and Metabolic Analysis Reveals a Complex Mechanism Involved in Drought Tolerance in Chickpea ( Cicer arietinum L .) Induced by PGPR and PGRs. Scientific reports 9:2097. 10.1038/s41598-01938702-8

Kim M, Kim HJ, Pak JH, Cho HS, Choi HK, Jung HW, Lee DH, and Chung Y. 2017. Overexpression of AtSZF2 from Arabidopsis Showed Enhanced Tolerance to Salt Stress in Soybean. Plant breeding and biotechnology 5:1-15. 10.9787/PBB.2017.5.1.1

Kloepper J, and Schroth M. 1978. Plant growth promoting rhizobacteria on radishes. In: phytobacteriologie Sdpve, editor Proceedings of the 4th International Conference on Plant Pathogenic Bacteria Tours, France: Gilbert-Clarey 2:879-882.

Kuljanabhagavad T, Thongphasuk P, Chamulitrat W, and Wink M. 2008. Triterpene saponins from Chenopodium quinoa Willd. Phytochemistry 69. 10.1016/j.phytochem.2008.03.001

Kuzyakov Y, and Razavi BS. 2019. Rhizosphere size and shape: Temporal dynamics and spatial stationarity. Soil Biology \& Biochemistry 135:343-360. 10.1016/j.soilbio.2019.05.011

Peer) reviewing PDF | (2020:08:52442:1:1:NEW 26 Nov 2020) 
369

370

371

372

373

374

375

376

377

378

379

380

381

382

383

384

385

386

387

388

389

390

391

392

393

394

395

396

397

398

399

400

401

402

403

404

405

406

407

408

409

410

411

412

413

414

415

416

417

418

419

Liao P, Liu D, Xu T, Yang Y, and Cui X. 2017. Soil water stress attenuate the growth and development but enhance the saponin synthesis of Panax notogesing during flowering stage. Industrial Crops and Products 108:95-105. 10.1016/j.indcrop.2017.05.052

Liu S, and Jiang Y. 2010. Identification of differentially expressed genes under drought stress in perennial ryegrass. Physiologia Plantarum 139:375-387. 10.1111/j.1399-3054.2010.01374.x

Liu W, Hou J, Wang Q, Ding L, and Luo Y. 2014. Isolation and characterization of plant growthpromoting rhizobacteria and their effects on phytoremediation of petroleum-contaminated salinealkali soil. Chemosphere 117:303-308. 10.1016/j.chemosphere.2014.07.026

Liu X, Zhu H, Wang L, Bi S, Zhang Z, Meng S, Zhang Y, Wang H, Song C, and Ma F. 2019. The effects of magnetic treatment on nitrogen absorption and distribution in seedlings of Populus $\times$ euramericana 'Neva' under $\mathrm{NaCl}$ stress. Scientific reports 9:10025-10025. $10.1038 / \mathrm{s} 41598-019-45719-6$

Mayak S, Tirosh T, and Glick BR. 2004. Plant growth-promoting bacteria confer resistance in tomato plants to salt stress. Plant Physiology and Biochemistry 42:565-572. 10.1016/j.plaphy.2004.05.009

Muhammad T, Zhang J, Ma Y, Li Y, Zhang F, Zhang Y, and Liang Y. 2019. Overexpression of a Mitogen-Activated Protein Kinase SIMAPK3 Positively Regulates Tomato Tolerance to Cadmium and Drought Stress. Molecules (Basel, Switzerland) 24:556. $10.3390 /$ molecules 24030556

Munns R, and Tester M. 2008. Mechanisms of salinity tolerance. Annual Review of Plant Biology 59:651681. 10.1146/annurev.arplant.59.032607.092911

Navruz-Varli S, and Sanlier N. 2016. Nutritional and health benefits of quinoa ( Chenopodium quinoa Willd.). Journal of Cereal Science 69.

Panta S, Flowers TJ, Lane PA, Doyle RB, Haros G, and Shabala S. 2014. Halophyte agriculture: Success stories. Environmental and Experimental Botany 107:71-83. 10.1016/j.envexpbot.2014.05.006

Safdarian M, Askari H, Shariati JV, and Nematzadeh G. 2019. Transcriptional responses of wheat roots inoculated with Arthrobacter nitroguajacolicus to salt stress. Scientific reports 9:1-12. $10.1038 / \mathrm{s} 41598-018-38398-2$

Schleiff U. 2008. Analysis of Water Supply of Plants Under Saline Soil Conditions and Conclusions for Research on Crop Salt Tolerance. Journal of Agronomy and Crop Science 194:1-8.

Su A, Niu S, Liu Y, He A, Zhao Q, Pare PW, Li M, Han Q, Khan SA, and Zhang J. 2017. Synergistic Effects of Bacillus amyloliquefaciens (GB03) and Water Retaining Agent on Drought Tolerance of Perennial Ryegrass. International Journal of Molecular Sciences 18:2651. 10.3390/ijms 18122651

Tisarum R, Theerawitaya C, Samphumphuang T, Polispitak K, Thongpoem P, Singh HP, and Cha-Um S. 2020. Alleviation of Salt Stress in Upland Rice (Oryza sativa L. ssp. indica cv. Leum Pua) Using Arbuscular Mycorrhizal Fungi Inoculation. Frontiers in Plant Science 11:348-348. $10.3389 /$ fpls. 2020.00348

Trivedi P, Leach JE, Tringe SG, Sa T, and Singh BK. 2020. Plant-microbiome interactions: from community assembly to plant health. Nature Reviews Microbiology 18:607-621. 10.1038/s41579020-0412-1

Szakiel A, Paczkowski C, and Henry M. 2011. Influence of environmental abiotic factors on the content of saponins in plants. Phytochemistry Reviews 10:471-491. 10.1007/s11101-010-9177-x

Tiwari S, and Lata C. 2018. Heavy Metal Stress, Signaling, and Tolerance Due to Plant-Associated Microbes: An Overview. Frontiers in Plant Science 9:452-452. 10.3389/fpls.2018.00452

Upadhyay SK, and Singh DP. 2015. Effect of salt - tolerant plant growth - promoting rhizobacteria on wheat plants and soil health in a saline environment. Plant Biology 17:288-293. 10.1111/plb.12173

Vardharajula S, Ali SZ, Grover M, Reddy G, and Bandi V. 2011. Drought-tolerant plant growth promoting Bacillus spp.: effect on growth, osmolytes, and antioxidant status of maize under drought stress. Journal of Plant Interactions 6:1-14. 10.1080/17429145.2010.535178

Peer) reviewing PDF | (2020:08:52442:1:1:NEW 26 Nov 2020) 
420

421

422

423

424

425

426

427

428

429

430

431

432

433

434

435

436

437

438

439

440

441

442

443

444

445

446

447

Wang H-M, Xiao X-R, Yang M-Y, Gao Z-L, Zang J, Fu X-M, and Chen Y-H. 2014. Effects of salt stress on antioxidant defense system in the root of Kandelia candel. Botanical studies 55:57-57. 10.1186/s40529-014-0057-3

Xi M, Hai C, Tang H, Chen M, Fang K, and Liang X. 2008. Antioxidant and antiglycation properties of total saponins extracted from traditional Chinese medicine used to treat diabetes mellitus. Phytotherapy Research 22:228-237.

Yang A, Akhtar SS, Iqbal S, Amjad M, Naveed M, Zahir ZA, and Jacobsen S. 2016. Enhancing salt tolerance in quinoa by halotolerant bacterial inoculation. Functional Plant Biology 43:632-642.

Yang A, Akhtar SS, Iqbal S, Qi Z, Alandia G, Saddiq MS, and Jacobsen SE. 2018. Saponin seed priming improves salt tolerance in quinoa. Journal of Agronomy and Crop Science 204. $10.1111 /$ jac. 12229

Yu J, Zhao W, Tong W, He Q, Yoon M, Li F, Choi B, Heo E, Kim K, and Park Y. 2018. A genome-wide association study reveals candidate genes related to salt tolerance in rice (oryza sativa) at the germination stage.. International Journal of Molecular Sciences 19. 10.3390/ijms19103145

Zeng Q, Wu X, and Wen X. 2016. Effects of Soluble Phosphate on Phosphate-Solubilizing Characteristics and Expression of gcd Gene in Pseudomonas frederiksbergensis JW-SD2. Current Microbiology 72:198-206. 10.1007/s00284-015-0938-z

Zhang Z, He K, Zhang T, Tang D, Li R, and Jia S. 2019. Physiological responses of Goji berry (Lycium barbarum L.) to saline-alkaline soil from Qinghai region, China. Scientific reports 9:1205712057. 10.1038/s41598-019-48514-5

Zhao Q, Wu Y-N, Fan Q, Han Q-Q, Pare PW, Xu R, Wang Y-Q, Wang S-M, and Zhang J-L. 2016. Improved Growth and Metabolite Accumulation in Codonopsis pilosula (Franch.) Nannf. by Inoculation of Bacillus amyloliquefaciens GB03. Journal of Agricultural and Food Chemistry 64:8103-8108. 10.1021/acs.jafc.6b03390

Zheng Y, Zhang H, Deng X, Liu J, and Chen H. 2017. The relationship between vacuolation and initiation of PCD in rice (Oryza sativa) aleurone cells. Scientific reports 7:41245. $10.1038 /$ srep41245

PeerJ reviewing PDF | (2020:08:52442:1:1:NEW 26 Nov 2020) 


\section{Figure 1}

Effect of M30-35 on the growth of $C$. quinoa under salt treatments $(0,150$ and $300 \mathrm{mM}$ of $\mathrm{NaCl}$ ).

$A$ is the shoot dry weight of quinoa after $7 d ; B$ is the shoot dry weight of $C$. quinoa after $21 d$;

$\mathrm{C}$ is the $\mathrm{NaCl}$ treatment for $7 \mathrm{~d}$, and root dry weight; $\mathrm{D}$ is the $\mathrm{NaCl}$ treatment for $21 \mathrm{~d}$, and root dry weight. The values are the means, and the bars are standard errors $(n=6)$. Columns with different letters indicate significant differences among treatments at $P<0.05$ (ANOVA and Duncan's multiple comparison test); * refers to the significant difference between the LB and M30-35 mediums $(P<0.05)$. 

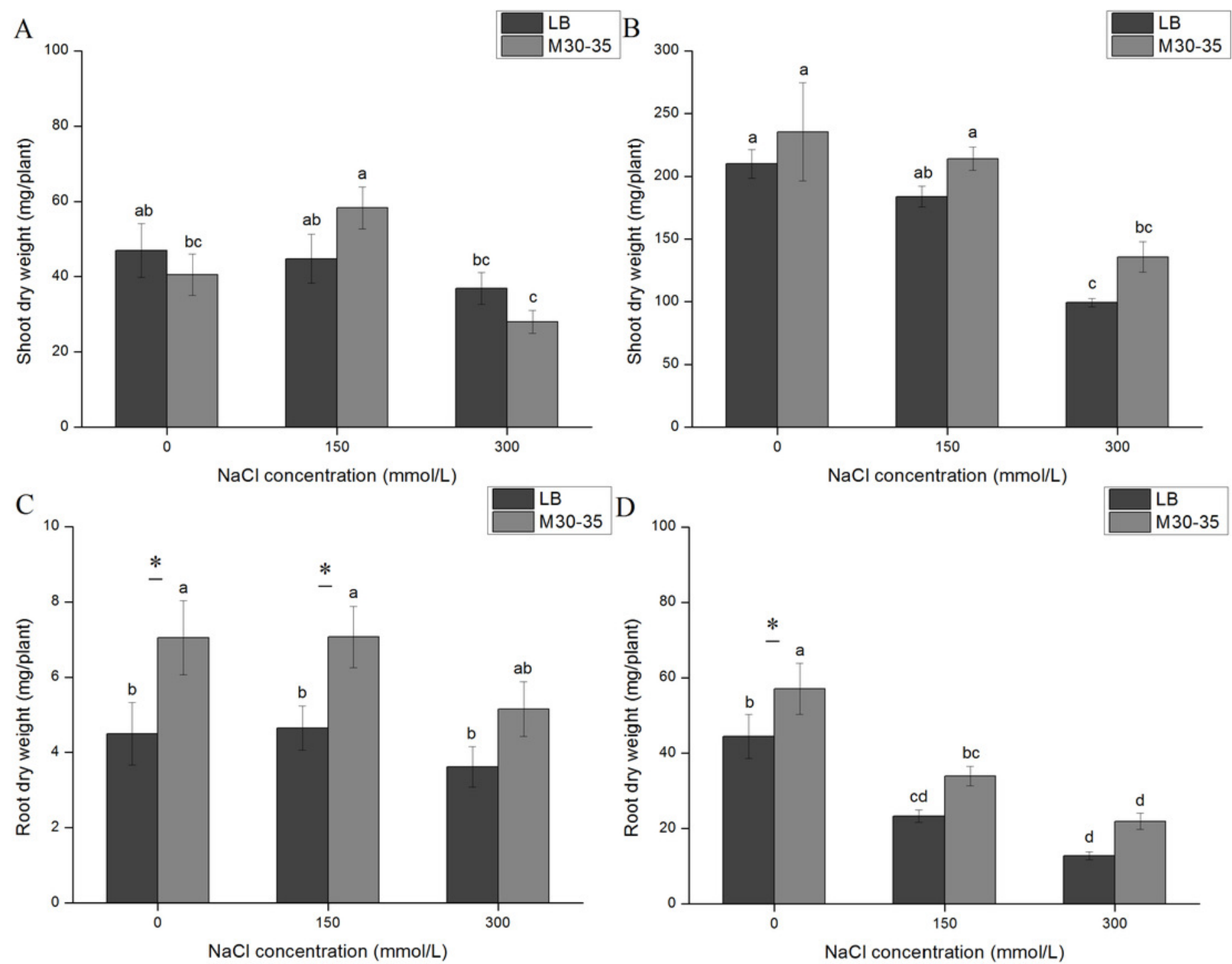


\section{Figure 2}

Effects of M30-35 on the leaf chlorophyll a content (A and B) and leaf chlorophyll b content $(C$ and $D)$ of $C$. quinoa under salt stress $(0,150$, and $300 \mathrm{mM}$ of $\mathrm{NaCl})$.

$A$ and $\mathrm{C}$ are $\mathrm{NaCl}$ treatments for $7 \mathrm{~d} . \mathrm{B}$ and $\mathrm{D}$ are $\mathrm{NaCl}$ treatments for $21 \mathrm{~d}$. The values are means, and the bars are standard errors $(n=6)$. Columns with different letters indicate the significant differences among treatments at $P<0.05$ (ANOVA and Duncan's multiple comparison test); * refers to the significant difference between the LB and M30-35 mediums $(P<0.05)$. ** refers to the difference between the LB and M30-35 mediums $(P<0.01)$.
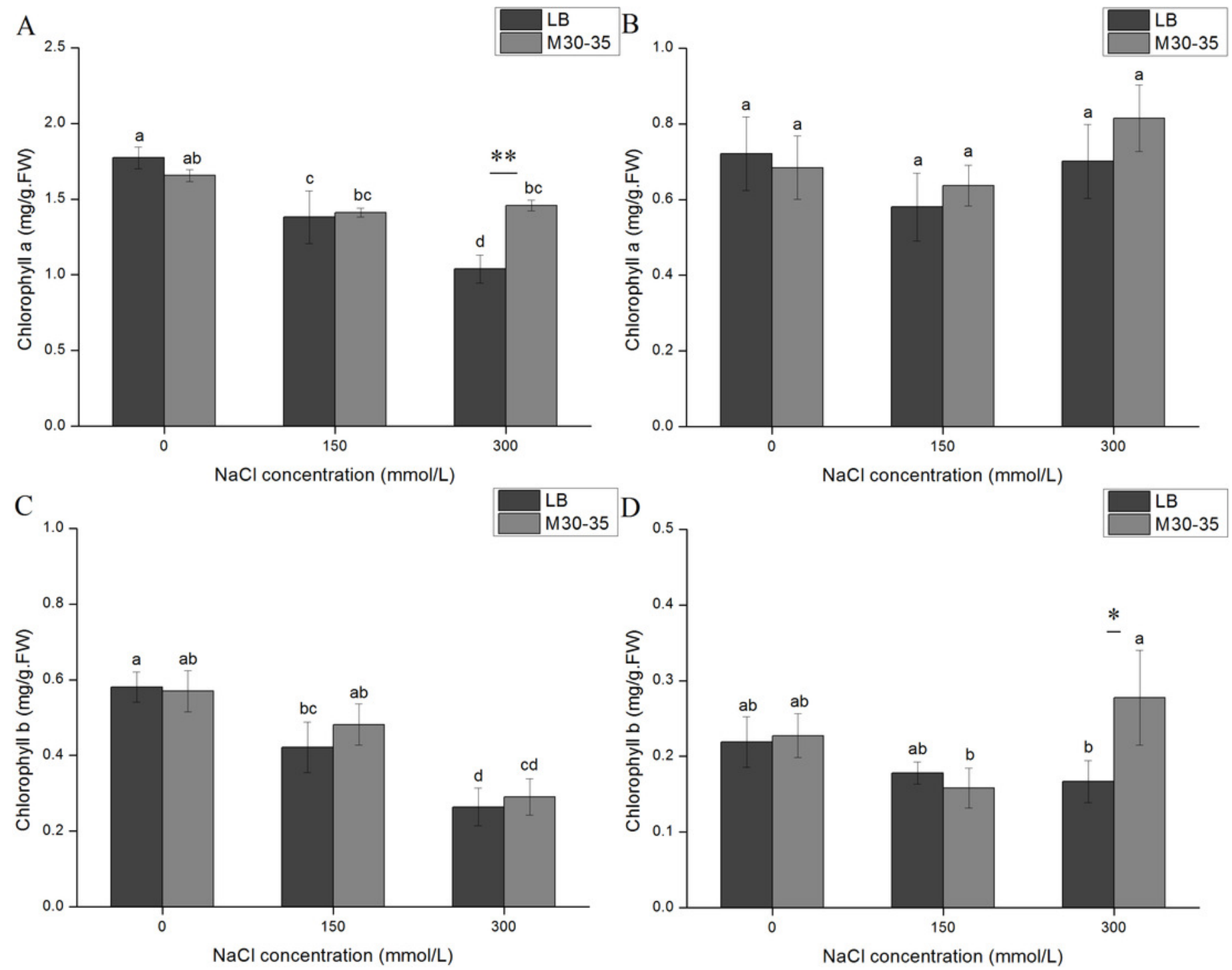


\section{Figure 3}

Effects of M30-35 on the root activity of $C$. quinoa under salt treatment $(0,150$, and 300 $\mathrm{mM}$ of $\mathrm{NaCl}$ ).

$A$ is the root activity of plants measured after $7 d ; B$ is the root activity of plants measured after $21 d$. The values are the means and the bars indicate the SEs $(n=3)$. Columns with different letters indicate significant differences among treatments at $P<0.05$ (ANOVA and Duncan's multiple comparison test). * refers to the significant difference between the LB and M30-35 mediums $(P<0.05)$.
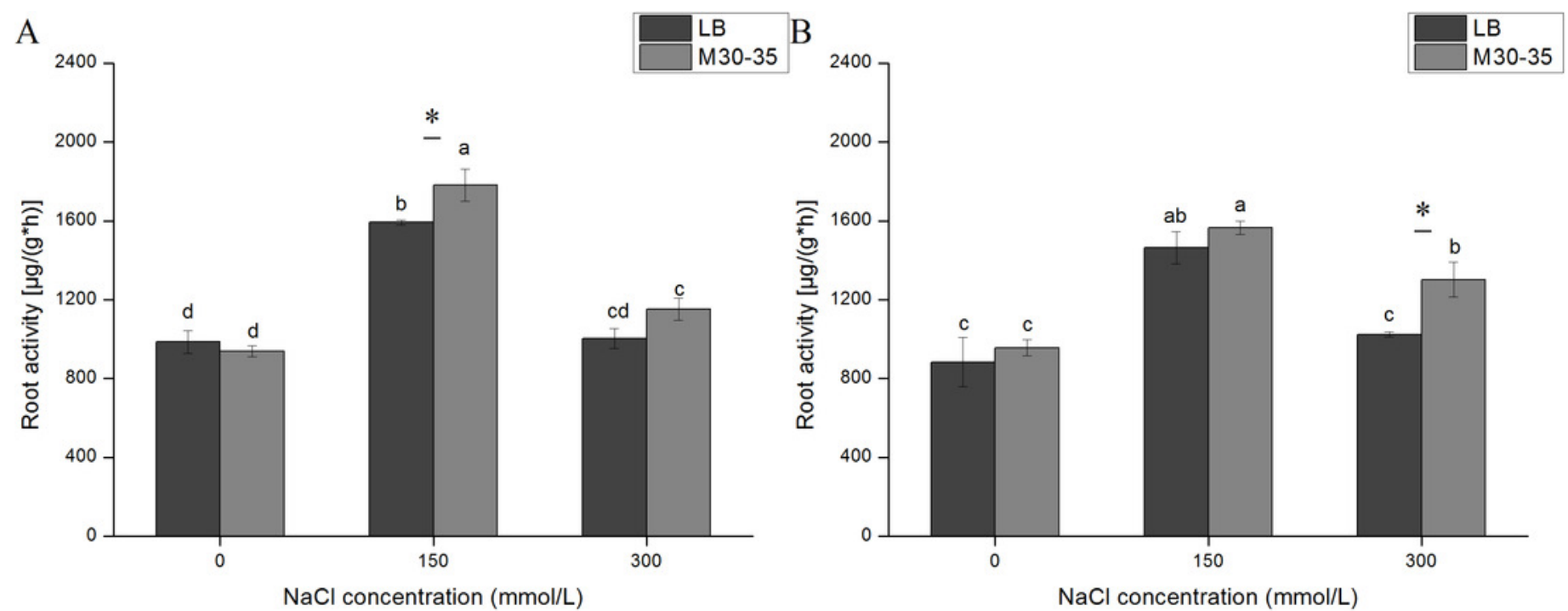


\section{Figure 4}

Comparison of the $\mathrm{P}$ content in plants under salt treatments $(0,150$, and $300 \mathrm{mM}$ of $\mathrm{NaCl})$.

$A$ is the total P content of plants measured after $7 d$; $B$ is the total $P$ content of plants measured after $21 \mathrm{~d}$. The values are the means and the bars indicate the SEs $(n=3)$. Columns with different letters indicate a significant difference among treatments at $P<0.05$ (ANOVA and Duncan's multiple comparison test). * refers to the significant difference between the LB and M30-35 mediums $(P<0.05)$.
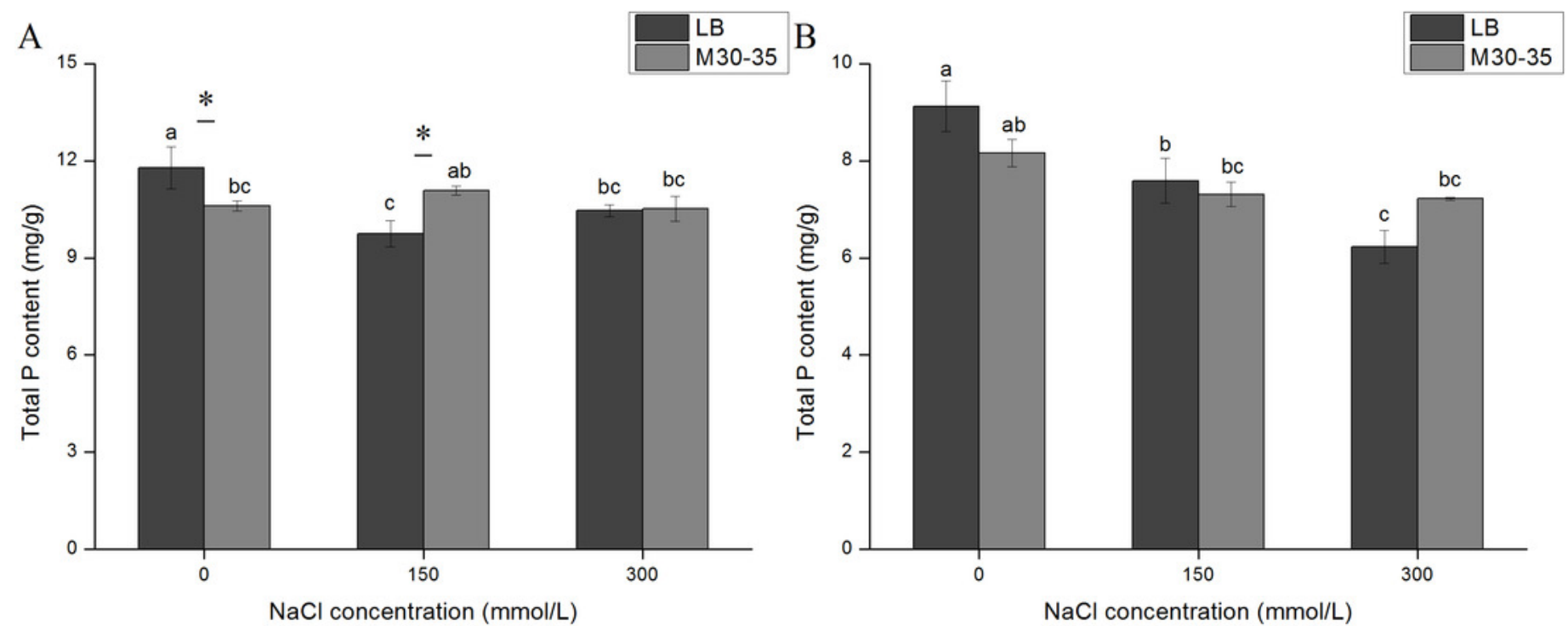


\section{Figure 5}

Effects of M30-35 on the content of saponins in $C$. quinoa under salt treatment $(0,150$, and $300 \mathrm{mM}$ of $\mathrm{NaCl}$ ).

The values are means and the bars indicate the SEs $(n=3)$. Columns with different letters indicate a significant differences among treatments at $P<0.05$ (ANOVA and Duncan's multiple comparison test). * refers to the significant difference between the LB and M30-35 mediums $(P<0.05)$.

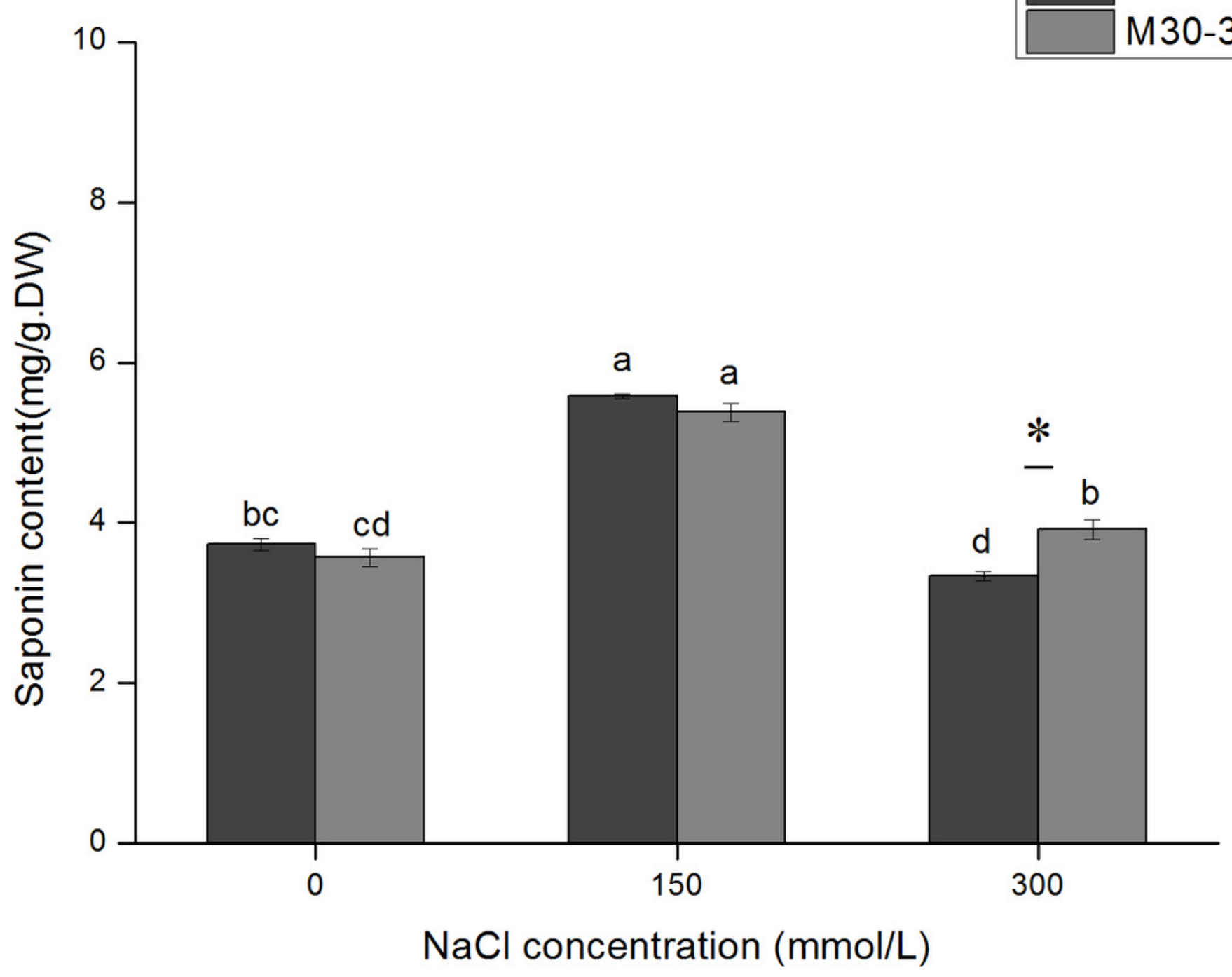

